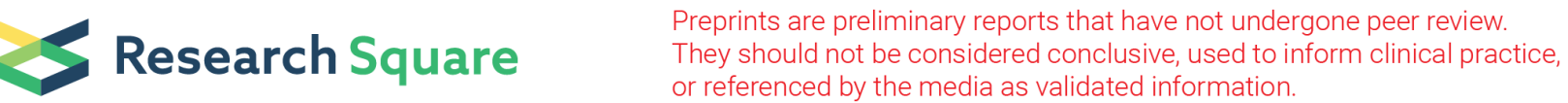

\section{Development and Validation of a Novel Predictive Nomogram for Disease Progression of COVID-19: A Multicenter Retrospective Cohort Study of 4,086 Cases in China}

\section{Li Li}

Third Military Medical University Daping Hospital

\section{Shen Li}

Chongqing Medical University

\section{Xiaohua Zhang}

Third Military Medical University Daping Hospital

\section{Lixia Cheng}

Third Military Medical University Daping Hospital

\section{Qing Mao}

Third Military Medical University Southwest Hospital

Qi Li

Third Military Medical University Second Affiliated Hospital: Xinqiao Hospital

\section{Xiaoyu Fang}

Third Military Medical University: Army Medical University

\section{Zhujun Li}

Third Military Medical University Daping Hospital

\section{Bin Wang}

Third Military Medical University Second Affiliated Hospital: Xinqiao Hospital

\section{Songtao Zhao}

Third Military Medical University Southwest Hospital

\section{Yi Zhou}

Third Military Medical University Second Affiliated Hospital: Xinqiao Hospital

\section{Kaijun Liu}

Third Military Medical University Daping Hospital

\section{Chuanwei Li}

Third Military Medical University Daping Hospital

\section{Chao Hou}

Third Military Medical University Daping Hospital

\section{Fenfen Sun}

Third Military Medical University Daping Hospital 


\section{Qidi Sun}

Third Military Medical University: Army Medical University

\section{Mengxia Li}

Third Military Medical University Daping Hospital

\section{Fusheng Wang}

5th Medical Center of Chinese PLA General Hospital

\section{Sibing Zhang}

Chinese PLA General Hospital

\section{Dixiong $\mathrm{Xu}$}

Third Military Medical University: Army Medical University

\section{Xiangyu Ma}

Third Military Medical University: Army Medical University

\section{Guoqiang Cao ( $\square$ cgq1963@163.com )}

Third Military Medical University Daping Hospital https://orcid.org/0000-0001-8648-7654

\section{Research}

Keywords: COVID-19, SARS-Cov-2, nomogram, severity, progression

Posted Date: October 26th, 2020

DOI: https://doi.org/10.21203/rs.3.rs-96165/v1

License: (c) (i) This work is licensed under a Creative Commons Attribution 4.0 International License. Read Full License 


\section{Abstract}

Aim: Coronavirus disease 2019 (COVID-19) has caused an unprecedented healthcare crisis. We aim to develop and validate a nomogram for predicting disease progression based on a large cohort of hospitalized COVID-19 patients.

Methods: This is a multicenter retrospective cohort study, with a total of 4,086 hospitalized COVID-19 patients enrolled from two hospitals in Wuhan, China between February $3^{\text {rd }}$ and $A p r 10^{\text {th }}$. Nomogram was developed based on a cohort of 3, 022 patients from one hospital, and externally validated in another cohort of 1,064 patients from the other hospital. The calibration was assessed by a calibration plot and the HL test to evaluate the goodness of fit, and the Area under the ROC Curve (AUROC) as a measure of discriminative ability.

Results: Six independent predictors, including age, dyspnea, platelet count, lactate dehydrogenase, Ddimer and cardiovascular disease, were finally identified for construction of the nomogram for predicting disease progression of COVID-19 patients during hospitalization. The AUROC was 0.877 and 0.817 for development cohort and validation cohort, respectively. The calibration plots AND Hosmer-Lemeshow test showed optimal agreement between nomogram prediction and actual observation. The decision curve analysis showed the performance of the nomograms were better than all univariable models, and had greater net benefit. Next, a predictive nomogram for disease severity on admission was formulated and the six independent factors used were similar to that of the nomogram for disease progression, which indicates that those factors play important roles in determining disease severity and the risk of disease progression.

Conclusion: In the current study, a nomogram was developed based on generally readily available variables at hospital admission to help predict disease progression of COVID-19.

\section{Background}

In December 2019, the ongoing coronavirus disease 2019 (COVID-19) broke out in Wuhan, China and soon spread all over the world, evolving into a pandemic. The number of patients confirmed to have the disease has exceeded more than 37,000,000 in countries, and more than 1, 070, 355 people have died from COVID-19 (up to 12 October 2020) ${ }^{1}$. COVID-19 patients exhibit a broad spectrum of disease severity and progression, with $81 \%$ showing mild, moderate or no symptoms; $14 \%$ showing severe symptoms and $5 \%$ experiencing critical disease and high risk of mortality ${ }^{2}$. Despite a relative low death rate, some patients with COVID-19 progressed rapidly and increasing respiratory support were given, including invasive mechanical ventilation. Identifying those patients at risk could help clinicians in triaging patients when allocating limited healthcare resources.

A quick index scoring system may help quickly identify hospitalized COVID-19 patients at high risk for worse outcome. Previous studies have found that several factors are associated with progression to severe or critical disease, including advanced age, comorbidities, hyperactivation of the immune system, 
sex, and other factors, and risk score system were developed ${ }^{3-7}$. However, integration of those risk factors, especially in a nomogram, is still lacking. As pointed out in a recent systemic review, a total of 8 prognostic models have been proposed to predict progression to severe or critical state, and another 23

models for prediction of mortality ${ }^{8}$. However, these models were considered at high risk of bias due to non-representative selection of control patients, high risk of model overfitting, vague reporting and other reasons ${ }^{8}$.

We therefore conducted a multicenter, retrospective cohort study involving 4,086 in-hospital COVID-19 patients. Two nomograms predicting disease severity at admission and disease progression were successfully developed in a development cohort of 3022 patients and validated in an external cohort of 1064 patients. The nomogram performed well by the area under receiver operating curve (AUROC) for discrimination and calibration plot together with Hosmer-Lemeshow $(\mathrm{HL})$ test for calibration. Decision curve analysis (DCA) finally supported the greater net benefit of the predictive models. Therefore, the current study established a simple and effective tool to help clinicians identify patients who are easy to deteriorate into critical cases and therefore optimizing treatment strategies, reducing mortality, and relieving the medical pressure.

\section{Methods}

\section{Study Population and Design}

This multicenter retrospective cohort study included a total of 3,022 COVID-19 cases recruited between February 3rd and Apr 10th from Wuhan Huoshenshan Hospital (used as development cohort), and 1,064 COVID-19 cases recruited between February 19th and Marth 18th from Taikang-Tongji hospital with relevant epidemiological and clinical data (used as validation cohort). The diagnosis of COVID-19 patients was based on the World Health Organization interim guidance. ${ }^{9}$ The nasal and pharyngeal swab specimens were collected for extracting SARS-Cov-2 RNA from patients suspected of having COVID-19 infection. Real-time reverse transcription polymerase chain reaction assay was then implemented for the laboratory confirmation of the SARS-Cov-2 virus. The epidemiological data was telephone-interviewed using a uniformed questionnaire by two trained physicians. The clinical symptoms and signs, laboratory, progression and outcome information were extracted from the electronic medical records. We double entered and validated the data using EpiData (version 3.1, EpiData Association, Odense, Denmark) software, and disputes were arbitrated by the expert committees composed of experts of respiratory and critical care medicine, radiograph, and epidemiology. The study protocol was approved by the ethics committee of Wuhan Huoshenshan Hospital (HSSLL024) and Taikang-Tongji hospital (TKTJKY2020146), and the informed consent was waived due to dealing with urgent public health concerns.

\section{Study Outcomes and Definitions}


Disease progression of COVID-19 was evaluated as the primary endpoint in the current study, and disease severity was evaluated as the secondary endpoint. The disease progression consisted of severity progression, admission to an intensive care unit (ICU), the use of mechanical ventilation, or death. The severity of COVID-19 at admission was determined according to "Guidance for COVID-19 Prevention, Control, Diagnosis and Management" by National Health Commission of the People's Republic of China, which was divided into four categories: mild, ordinary, severe and critical. ${ }^{10}$ Mild, ordinary were categized as non-severe, while severe and critical were categized as severe. Fever was defined as an axillary temperature of $37.3^{\circ} \mathrm{C}$ or higher.

\section{Statistical analysis}

All statistical analyses were conducted using the R software version 4.0.0 (Institute for Statistics and Mathematics, Vienna, Austria). The reported statistical significance levels were all 2-sided, and a level of statistical significance set at $<0.05$ was considered statistically significant. Categorical variables were described using frequency rates and percentages, while continuous variables were described using the median/interquartile range (IQR) values. The missing values of all potential predictors (missing rate of less than $10.0 \%$ ) were imputed by expectation-maximization (EM) method. Logistic regression analysis was adopted for the estimation of odds ratio (OR) and corresponding confidence interval $(\mathrm{Cl})$ of candidate variables. The following procedures were implemented to screen the candidate variables in the development cohort. First, univariate logistic regression analysis was used to screen the potential prognostic factors which reached a $\mathrm{P}$ value of less than 0.05 . Then, the independent risk factors were derived from a backward stepdown selection process in multivariate logistic regression model. Finally, a predictive nomogram was formulated based on the results of multivariate analysis according to the Akaike information criterion and the random forest procedure. ${ }^{11,12}$ Nomogram calibration was assessed by a calibration plot and the HL test to evaluate the goodness of fit, and the AUROC as a measure of discriminative ability. The nomograms were applied to an independent cohort (1,064 COVID-19 cases from Taikang-Tongji hospital) to validate and evaluate the prediction efficacy. During the validation of the prognostic nomogram, the total points of each patient in the validation cohort were calculated according to the established nomogram, then logistic regression in this cohort was performed using the total points as a factor, and finally the AUROC and calibration plot were derived based on the regression analysis. Finally, DCA was used to evaluate the net benefit of the predictive models.

\section{Results}

\section{Characteristics of Development and Validation Cohorts}

The demographic and clinical characteristics of the COVID-19 patients in the development cohort and the validation cohort were presented in Table 1. Of the total 4,086 COVID-19 cases, $28.3 \%(N=1,156)$ were categorized as severe, and 6.7\% $(\mathrm{N}=273)$ reached the endpoint of disease progression. Fever $(70.6 \%)$, 
cough $(69.2 \%)$, and fatigue $(5=54.7 \%)$ ranked the top three symptoms, while hypertension $(31.7 \%)$, diabetes (14.5\%), and cardiovascular disease (10.5\%) ranked the top three coexisting disorders. 
Table 1

Demographic and clinical characteristics of the included COVID-19 patients

\begin{tabular}{|c|c|c|c|}
\hline Variables & Total $(\mathrm{N}=4086)$ & $\begin{array}{l}\text { Development cohort }(\mathrm{N}= \\
3022)\end{array}$ & $\begin{array}{l}\text { Validation cohort }(\mathrm{N}= \\
\text { 1064) }\end{array}$ \\
\hline Gender, male & $2043(50.0 \%)$ & $1541(51.0 \%)$ & $502(47.2 \%)$ \\
\hline $\begin{array}{l}\text { Age (Years old), median } \\
\text { (IQR) }\end{array}$ & $61(50-69)$ & $60(49-68)$ & $62(51-71)$ \\
\hline $0-14$ & $3(0.1 \%)$ & $2(0.1 \%)$ & $1(0.1 \%)$ \\
\hline $15-49$ & 1003 (24.5\%) & $761(25.2 \%)$ & $242(22.7 \%)$ \\
\hline $50-64$ & 1505 (36.8\%) & $1155(38.2 \%)$ & $350(32.9 \%)$ \\
\hline$\geq 65$ & $1575(38.5 \%)$ & $1104(36.5 \%)$ & $471(44.3 \%)$ \\
\hline \multicolumn{4}{|l|}{ Severity at admission } \\
\hline Non-severe & $2930(71.7 \%)$ & $2194(72.6 \%)$ & $736(69.2 \%)$ \\
\hline Mild & $48(1.2 \%)$ & $31(1.0 \%)$ & $17(1.6 \%)$ \\
\hline Ordinary & $2882(70.5 \%)$ & $2163(71.6 \%)$ & $719(67.6 \%)$ \\
\hline Severe & $1156(28.3 \%)$ & $828(27.4 \%)$ & $328(30.8 \%)$ \\
\hline Severe & 1076 (26.3\%) & $790(26.1 \%)$ & $286(26.9 \%)$ \\
\hline Critical & $80(2.0 \%)$ & $38(1.3 \%)$ & $42(3.9 \%)$ \\
\hline Disease progression & $273(6.7 \%)$ & $162(5.4 \%)$ & $111(10.4 \%)$ \\
\hline Death & $98(2.4 \%)$ & $69(2.3 \%)$ & $29(2.7 \%)$ \\
\hline ICU admission & $139(3.4 \%)$ & $107(3.5 \%)$ & $32(3.0 \%)$ \\
\hline Mechanical ventilation & $120(2.9 \%)$ & $85(2.8 \%)$ & $35(3.3 \%)$ \\
\hline Severity progression & $142(3.5 \%)$ & $84(2.8 \%)$ & $58(5.5 \%)$ \\
\hline Respiratory rate (times) & $20(18-21)$ & $20(19-21)$ & $16(11-23)$ \\
\hline \multicolumn{4}{|l|}{ Symptoms - no. (\%) } \\
\hline Fever & $2885(70.6 \%)$ & $2222(73.5 \%)$ & $663(62.3 \%)$ \\
\hline Cough & $2827(69.2 \%)$ & 2187 (72.4\%) & $640(60.2 \%)$ \\
\hline Fatigue & 2237 (54.7\%) & $1761(58.3 \%)$ & $476(44.7 \%)$ \\
\hline Anorexia & 2145 (52.5\%) & $1691(56.0 \%)$ & 454 (42.7\%) \\
\hline Short breath & $1686(41.3 \%)$ & 1466 (48.5\%) & $220(20.7 \%)$ \\
\hline
\end{tabular}

All continuous variables were presented using the median/interquartile range (IQR) values. 


\begin{tabular}{|c|c|c|c|}
\hline Variables & Total $(\mathrm{N}=4086)$ & $\begin{array}{l}\text { Development cohort }(\mathrm{N}= \\
\text { 3022) }\end{array}$ & $\begin{array}{l}\text { Validation cohort }(\mathrm{N}= \\
\text { 1064) }\end{array}$ \\
\hline Myalgia & $1093(36.7 \%)$ & $1004(33.2 \%)$ & $89(8.4 \%)$ \\
\hline Chest tight & $1179(28.9 \%)$ & $881(29.2 \%)$ & $298(28.0 \%)$ \\
\hline Expectoration & $728(17.8 \%)$ & $527(17.4 \%)$ & $201(18.9 \%)$ \\
\hline Diarrhea & $327(8.0 \%)$ & $186(6.2 \%)$ & $134(12.6 \%)$ \\
\hline Dyspnea & $268(6.6 \%)$ & $193(6.4 \%)$ & $82(7.7 \%)$ \\
\hline Sore throat & $227(5.6 \%)$ & $128(4.2 \%)$ & $99(9.3 \%)$ \\
\hline Nausea & $121(3.0 \%)$ & $88(2.9 \%)$ & $33(3.1 \%)$ \\
\hline Headache & $104(2.5 \%)$ & $70(2.3 \%)$ & $34(3.2 \%)$ \\
\hline Vomiting & $104(2.5 \%)$ & $72(2.4 \%)$ & $32(3.0 \%)$ \\
\hline Dizziness & $107(2.6 \%)$ & $76(2.5 \%)$ & $31(2.9 \%)$ \\
\hline Chill & $94(2.3 \%)$ & $59(2.0 \%)$ & $35(3.3 \%)$ \\
\hline Hemoptysis & $26(0.6 \%)$ & $21(0.7 \%)$ & $5(0.5 \%)$ \\
\hline \multicolumn{4}{|l|}{$\begin{array}{l}\text { Coexisting disorders - no. } \\
(\%)\end{array}$} \\
\hline Hypertension & $1294(31.7 \%)$ & $920(30.4 \%)$ & $374(35.2 \%)$ \\
\hline Diabetes & $592(14.5 \%)$ & $528(14.2 \%)$ & $164(15.4 \%)$ \\
\hline Coronary heart disease & $427(10.5 \%)$ & $210(6.9 \%)$ & $146(13.7 \%)$ \\
\hline Cardiovascular disease & $300(7.3 \%)$ & $281(9.3 \%)$ & $90(8.5 \%)$ \\
\hline Cerebrovascular disease & $215(5.3 \%)$ & $116(3.8 \%)$ & $22(2.1 \%)$ \\
\hline Tumor & $195(4.8 \%)$ & $77(2.5 \%)$ & $79(7.4 \%)$ \\
\hline Bronchitis & $175(4.3 \%)$ & $52(1.7 \%)$ & $86(8.1 \%)$ \\
\hline COPD & $123(3.0 \%)$ & $30(1.0 \%)$ & $28(2.6 \%)$ \\
\hline Respiratory disease & $92(2.3 \%)$ & $89(2.9 \%)$ & $15(1.4 \%)$ \\
\hline Chronic kidney disease & $81(2.0 \%)$ & $95(3.1 \%)$ & $29(2.7 \%)$ \\
\hline Chronic liver disease & $60(1.5 \%)$ & $193(6.4 \%)$ & $30(2.8 \%)$ \\
\hline \multicolumn{4}{|l|}{ Laboratory findings } \\
\hline White-cell count $\left(\times 10^{9} / \mathrm{L}\right)$ & $5.8(4.7-7.1)$ & $5.7(4.7-7.1)$ & $5.8(4.8-7.0)$ \\
\hline
\end{tabular}




\begin{tabular}{|c|c|c|c|}
\hline Variables & Total $(\mathrm{N}=4086)$ & $\begin{array}{l}\text { Development cohort }(\mathrm{N}= \\
\text { 3022) }\end{array}$ & $\begin{array}{l}\text { Validation cohort }(\mathrm{N}= \\
\text { 1064) }\end{array}$ \\
\hline Neutrophil count $\left(\times 10^{9} / \mathrm{L}\right)$ & $\begin{array}{l}3.52(2.68- \\
4.73)\end{array}$ & $3.520(2.70-4.68)$ & $3.51(2.66-4.89)$ \\
\hline $\begin{array}{l}\text { Lymphocyte count (x } \\
\left.10^{9} / \mathrm{L}\right)\end{array}$ & $\begin{array}{l}1.52(1.11- \\
1.92)\end{array}$ & $1.49(1.09-1.86)$ & $1.65(1.23-2.11)$ \\
\hline Monocyte count $\left(\times 10^{9} / \mathrm{L}\right)$ & $\begin{array}{l}0.45(0.35- \\
0.58)\end{array}$ & $0.43(0.34-0.54)$ & $0.53(0.41-0.69)$ \\
\hline Platelet count $\left(\times 10^{9} / \mathrm{L}\right)$ & $223(180-274)$ & $224(181-276)$ & $219(177-265)$ \\
\hline C-reactive protein $(\mathrm{mg} / \mathrm{l})$ & $\begin{array}{l}2.07(0.60- \\
8.66)\end{array}$ & $2.35(0.83-9.02)$ & $0.50(0.50-6.33)$ \\
\hline D-dimer, mg/L & $\begin{array}{l}0.41(0.19- \\
0.86)\end{array}$ & $0.42(0.21-0.90)$ & $0.24(0.10-0.68)$ \\
\hline $\begin{array}{l}\text { Alanine aminotransferase, } \\
\mathrm{U} / \mathrm{L}\end{array}$ & $\begin{array}{l}22.5(14.3- \\
37.2)\end{array}$ & $22.9(14.6-38.2)$ & $21.6(13.8-35.2)$ \\
\hline $\begin{array}{l}\text { Aspartate } \\
\text { aminotransferase, U/L }\end{array}$ & $\begin{array}{l}20.0(15.9- \\
26.9)\end{array}$ & $19.7(15.7-26.5)$ & $21.3(17.0-27.8)$ \\
\hline Albumin, $\mathrm{g} / \mathrm{L}$ & $\begin{array}{l}37.8(34.7- \\
40.4)\end{array}$ & $37.8(34.8-40.3)$ & $38.1(34.5-40.8)$ \\
\hline Total bilirubin, $\mu \mathrm{mol} / \mathrm{L}$ & $9.9(7.6-12.6)$ & $9.4(7.3-12.3)$ & $10.6(8.6-13.2)$ \\
\hline Blood glucose, $\mathrm{mmol} / \mathrm{L}$ & $\begin{array}{l}5.02(4.55- \\
5.77)\end{array}$ & $4.88(4.47-5.64)$ & $5.36(4.91-6.05)$ \\
\hline Urea, mmol/L & $\begin{array}{l}4.58(3.73- \\
5.55)\end{array}$ & $4.37(3.58-5.45)$ & $4.99(4.28-5.75)$ \\
\hline Creatinine, $\mu \mathrm{mol} / \mathrm{L}$ & $\begin{array}{l}62.2(52.7- \\
74.4)\end{array}$ & $64.3(55.0-75.7)$ & $54.7(45.3-68.0)$ \\
\hline $\begin{array}{l}\text { Lactate dehydrogenase, } \\
\mathrm{U} / \mathrm{L}\end{array}$ & $\begin{array}{l}171.5(144.7- \\
208.0)\end{array}$ & $180.2(150.3-226.1)$ & 171.5 (149.7-202.1) \\
\hline
\end{tabular}

\section{Development and validation of a predictive nomogram for disease progression of COVID-19}

We first constructed the nomogram for predicting disease progression of COVID-19 patients during hospitalization. Supplementary Table 1 presented the results of univariate logistic regression analysis for disease progression of COVID-19 in the development cohort. Six independent predictors were finally 
identified for the construction of the nomogram (Table 2, Fig. 1), including age (OR for per year increment: 1.02; 95\% Cls: 1.00-1.03; $\mathrm{P}=0.022$ ), dyspnea (OR: 4.59; 95\% Cls: 2.94-7.14; $\mathrm{P}<0.001$ ), platelet count (OR: 2.96; 95\% Cls: 1.93-4.49; P< 0.001), lactate dehydrogenase (OR: 6.92; 95\% Cls: 4.77-10.11; P<0.001), Ddimer (OR: 3.92; 95\% Cls: 2.25-7.25; P< 0.001), and cardiovascular disease (OR: 2.34; 95\% Cls: $1.48-$ 3.66; $\mathrm{P}<0.001)$. The AUROC $(0.877,95 \% \mathrm{Cl}: 0.847-0.908)$ showed good discriminating ability (Supplementary Fig. 1). The calibration plot showed an optimal agreement between the prediction by nomogram and actual observation (Supplementary Fig. 2), and verified by HL test $(P=0.348)$. For the external validation, the AUROC $(0.817,95 \% \mathrm{Cl}: 0.773-0.861)$ confirmed good discriminating ability (Supplementary Fig. 3). The calibration plot (Supplementary Fig. 4) and HL test ( $P=0.685$ ) showed an optimal agreement between the prediction by nomogram and actual observation. The DCA showed the performance of the nomogram was better than all univariable models, and had greater net benefit (Fig. 2).

Table 2

Variables included in the nomogram construction for severity at admission and disease progression of COVID-19 in the development cohort

\begin{tabular}{|lllll|}
\hline & \multicolumn{2}{l}{ Severity at admission } & \multicolumn{2}{l|}{ Disease progression } \\
\hline Variables & HR $(95 \% \mathrm{Cls})$ & P value & HR $(95 \% \mathrm{Cls})$ & P value \\
\hline Age, per year increment & $1.03(1.02-1.04)$ & $<0.001$ & $1.02(1.00-1.03)$ & 0.022 \\
\hline Dyspnea & $3.40(2.44-4.76)$ & $<0.001$ & $4.59(2.94-7.14)$ & $<0.001$ \\
\hline Cardiovascular disease & - & & $2.34(1.48-3.66)$ & $<0.001$ \\
\hline Platelet count, $<150 \times 10^{9} / \mathrm{L}$ & $1.41(1.09-1.83)$ & 0.009 & $2.96(1.93-4.49)$ & $<0.001$ \\
\hline D-dimer, $\geq 0.5 \mathrm{mg} / \mathrm{L}$ & $1.51(1.25-1.82)$ & $<0.001$ & $3.92(2.25-7.25)$ & $<0.001$ \\
\hline Blood glucose, $>6.1 \mathrm{mmol} / \mathrm{L}$ & $1.57(1.27-1.94)$ & $<0.001$ & - & \\
\hline Lactate dehydrogenase, $\geq 250 \mathrm{U} / \mathrm{L}$ & $2.75(2.18-3.47)$ & $<0.001$ & $6.92(4.77-10.11)$ & $<0.001$ \\
\hline
\end{tabular}

\section{Development and validation of a predictive nomogram for severity of COVID-19 at admission}

We next asked whether the factors used for predicting disease progression may also play a role in determining disease severity on admission. Therefore, we set up a nomogram for predicting disease severity. Supplementary Table 2 presented the results of univariate logistic regression analysis for severity of COVID-19 at admission in the development cohort. Six independent predictors were finally identified for the construction of the nomogram (Table 2, Fig. 3), including age (OR for per year increment: 1.03; 95\% Cls: 1.02-1.04; P<0.001), dyspnea (OR: 3.40; 95\% Cls: 2.44-4.76; $P<0.001$ ), platelet count 
(OR: 1.41; 95\% Cls: 1.09-1.83; P = 0.009), lactate dehydrogenase (OR: $2.75 ; 95 \%$ Cls: $2.18-3.47 ; \mathrm{P}<$ 0.001), D-dimer (OR: 1.51; $95 \%$ Cls: 1.25-1.82; P<0.001), and blood glucose (OR: 1.57; 95\% Cls: $1.27-$ 1.94; $\mathrm{P}<0.001)$. The AUROC $(0.726,95 \% \mathrm{Cl}: 0.705-0.746)$ showed good discriminating ability

(Supplementary Fig. 5). The calibration plot showed an optimal agreement between the prediction by nomogram and actual observation (Supplementary Fig. 6 ), and verified by HL test $(P=0.949)$. For the external validation, the AUROC $(0.783,95 \% \mathrm{Cl}: 0.753-0.812)$ confirmed good discriminating ability (Supplementary Fig. 7). The calibration plot (Supplementary Fig. 8) and HL test $(P=0.578$ ) showed an optimal agreement between the prediction by nomogram and actual observation. The DCA showed the performance of the nomogram was better than all univariable models, and had greater net benefit (Fig. 4).

\section{Discussion}

The current study established a nomogram for predicting COVID-19 disease progression in a relatively large cohort of 4, 086 patients. The model displayed well performance with satisfactory accuracy in both the development and external validation cohorts. The variables required for calculation of the nomogram are generally readily available at hospital admission, so the nomogram can be easily used by clinicians to estimate an individual hospitalized patient's disease severity and risk of disease progression during hospitalization.

A number of risk factors have been reported to be associated with COVID-19 severity and worse prognosis, including mortality. The impact of smoking on COVID-19 severity has been previously reported, showing that patients with any smoking history are vulnerable to severe COVID-19 and worse in-hospital outcomes $^{13}$. Besides, a history of cerebrovascular disease might constitute an important risk factor of unfavorable clinical course of COVID-1914. Most recently, it was reported that age, COPD, immunocompromised status, time from disease onset, troponin concentration, and acute kidney injury were independently associated with mortality ${ }^{5}$. In a cohort of 1113 patients, several risk factors were selected to develop a PREDI-CO score (points to each variable an individual risk score) to predict severe respiratory failure (SRF) among patients hospitalized with COVID-19 ${ }^{15}$. These risk factors included age $\geq$ 70 years, obesity, body temperature $\geq 38^{\circ} \mathrm{C}, \mathrm{RR} \geq 22 \mathrm{bpm}$, lymphocytes $\leq 900 / \mathrm{mm} 3$, creatinine $\geq$ $1 \mathrm{mg} / \mathrm{dl}$, C-reactive protein $\geq 10 \mathrm{mg} / \mathrm{dl}$, and lactate dehydrogenase $\geq 350 \mathrm{IU} / \mathrm{L}$. Another online risk calculator was developed to predict a patient's risk of developing critical illness (a composite measure of admission to the intensive care unit, invasive ventilation, or death) ${ }^{4}$. Taken together, the above-mentioned models were based on different populations, often with a small sample size, and different endpoints were selected. Therefore, great caution should be paid to application of those models in clinic.

The nomograms established in the current study have several strengths. First, we have a relatively large sample size, with more than four thousand patients enrolled. Second, it is of note that all patients in both development cohort and external validation cohort have reached the composite endpoints, which enables the current study population a sound representation of hospitalized COVID-19 patients. Third, the

Page $11 / 18$ 
nomograms performed well in external validation of an independent cohort, suggesting that it is potentially applicable in different populations. The current study also has several limitations. First, the current study is of retrospective nature and the two nomograms need further validation in prospective studies. Second, due to emergent conditions, some data are missing for certain variables. Third, the data for model development and validation are all from Wuhan, China, which could potentially limit the generalizability of those models in other areas of the world.

\section{Conclusion}

In this study, we developed a nomogram to predict the disease progression among patients with COVID19 based on variables commonly measured on admission to the hospital. Estimating the risk of disease progression could help identify patients who are more easily to develop critical illness, thus helping clinicians optimizing treatment strategies and use of medical resources.

\section{Abbreviations}

AUROC: Area under the ROC Curve

Cl: confidence interval

COVID-19: Coronavirus disease 2019

DCA: Decision curve analysis

HL: Hosmer-Lemeshow

IQR: interquartile range

OR: odds ratio

\section{Declarations}

\section{Ethics approval and consent to participate}

The study protocol was approved by the ethics committee of Wuhan Huoshenshan Hospital (HSSLL024) and Taikang-Tongji hospital (TKTJKY2020146).

\section{Consent for publication}

The informed consent was waived due to dealing with urgent public health concerns. All authors critically revised the manuscript for important intellectual content and gave final approval for the version to be published. 


\section{Availability of data and materials}

Datasets for this research are included in this paper (and its supplementary information files). Further details will be made available to researchers in contact with the corresponding author.

\section{Competing interests}

The authors declare that no potential conflicts of interest.

\section{Funding}

The present study was funded by Outstanding Youth Science Foundation of Chongqing (cstc2020jcyjjq0129), National Natural Science foundation of China (81672287) and the Science Foundation for Outstanding Young People of the Army Medical University (grant to Pro Xiangyu Ma and Li Li). The funders had no role in study design, data collection and analysis, decision to publish, or preparation of the manuscript.

\section{Authors' contributions}

CG and MX conceived and designed the study. MX, LL and LS drafted the paper and did the statistical analysis. All authors collected the data. All authors approved the final draft of the manuscript for publication.

\section{Acknowledgements}

We thank the patients who enrolled in this study, and the physicians, nurses, and secretaries who have taken part in this study for their important contributions.

\section{References}

1. WHO. WHO COVID-19 Dashboard. (2020).

2. Wu, Z. \& McGoogan, J.M. Characteristics of and Important Lessons From the Coronavirus Disease 2019 (COVID-19) Outbreak in China: Summary of a Report of 72314 Cases From the Chinese Center for Disease Control and Prevention. JAMA (2020).

3. Zhang, S., et al. Development and validation of a risk factor-based system to predict short-term survival in adult hospitalized patients with COVID-19: a multicenter, retrospective, cohort study. Crit Care 24, 438 (2020). 
4. Liang, W., et al. Development and Validation of a Clinical Risk Score to Predict the Occurrence of Critical Illness in Hospitalized Patients With COVID-19. JAMA Intern Med (2020).

5. Azoulay, E., et al. Increased mortality in patients with severe SARS-CoV-2 infection admitted within seven days of disease onset. Intensive Care Med (2020).

6. Hajifathalian, K., et al. Development and external validation of a prediction risk model for short-term mortality among hospitalized U.S. COVID-19 patients: A proposal for the COVID-AID risk tool. PLoS One 15, e0239536 (2020).

7. Torres-Macho, J., et al. The PANDEMYC Score. An Easily Applicable and Interpretable Model for Predicting Mortality Associated With COVID-19. J Clin Med 9(2020).

8. Wynants, L., et al. Prediction models for diagnosis and prognosis of covid-19 infection: systematic review and critical appraisal. BMJ 369, m1328 (2020).

9. Organization, W.H. Clinical management of severe acute respiratory infection when Novel coronavirus (nCoV) infection is suspected: interim guidance. (January 28, 2020).

10. Commission, H.C. The New Coronavirus Pneumonia Prevention and Control Guidelines of China. (2020).

11. Harrell, F.E., Jr., Lee, K.L. \& Mark, D.B. Multivariable prognostic models: issues in developing models, evaluating assumptions and adequacy, and measuring and reducing errors. Stat Med 15, 361-387 (1996).

12. Cutler, D.R., et al. Random forests for classification in ecology. Ecology 88, 2783-2792 (2007).

13. Reddy, R.K., et al. The effect of smoking on COVID-19 severity: A systematic review and metaanalysis. J Med Virol (2020).

14. Siepmann, T., et al. Association of history of cerebrovascular disease with severity of COVID-19. J Neurol (2020).

15. Bartoletti, M., et al. Development and validation of a prediction model for severe respiratory failure in hospitalized patients with SARS-Cov-2 infection: a multicenter cohort study (PREDI-CO study). Clin Microbiol Infect (2020).

\section{Figures}

\section{Figure 1}

Nomogram for disease progression of COVID-19 in the development cohort. To use the nomogram, an individual patient's value is located on each variable axis, and a line is drawn upward to determine the number of points received for each variable value. The sum of these numbers is located on the Total Points axis, and a line is drawn downward to the "Risk axes" to determine the likelihood of disease progression of COVID-19. 


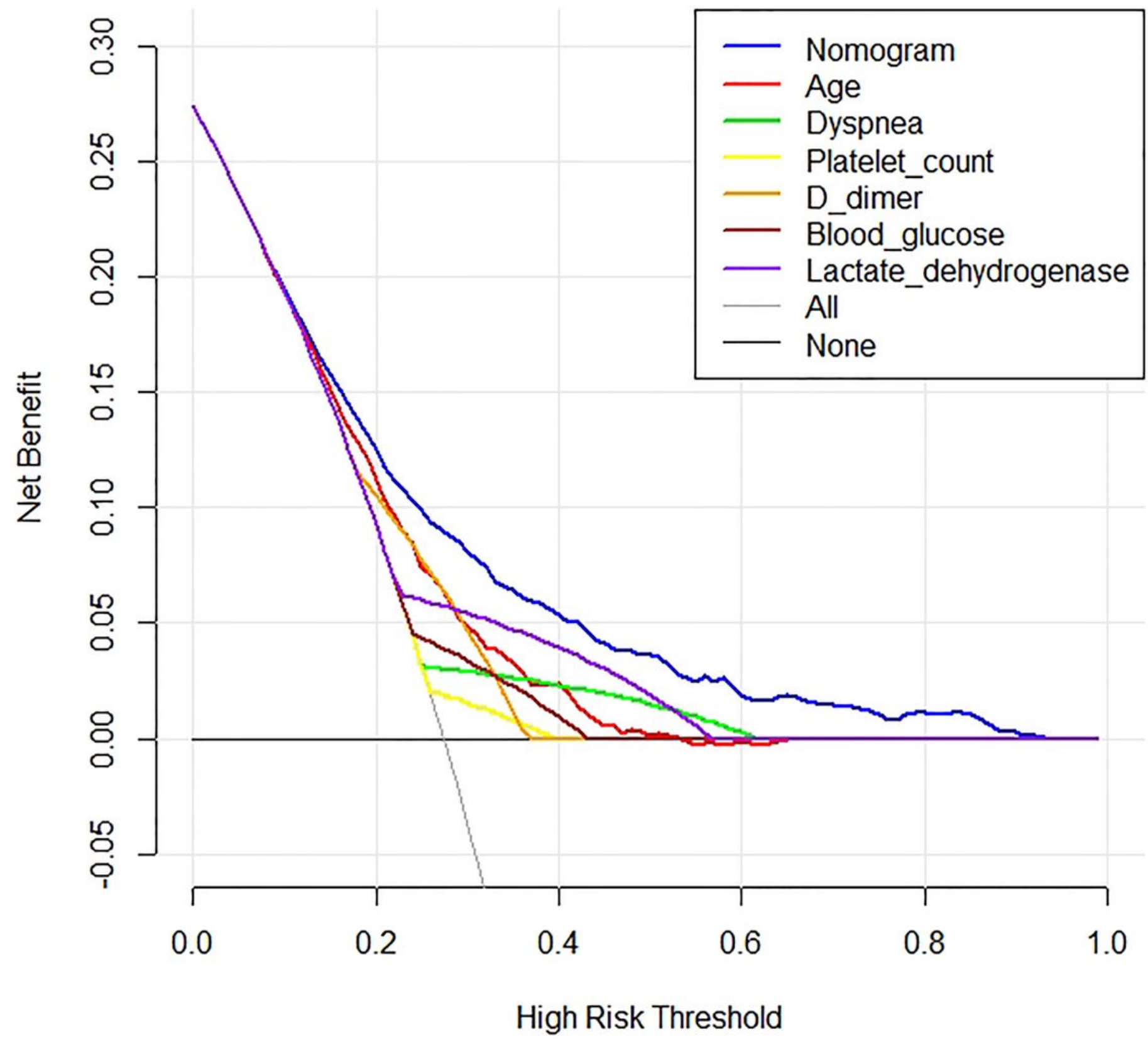

Figure 2

Decision curve analysis for disease progression of COVID-19. Decision curve analysis was performed to compare the performance of the nomogram with that of all univariable models. 


Points
Age
Cardiovascular_disease
Dyspnea
Platelet_count
D_dimer
Lactate_dehydrogenase
Total Points
Risk

Risk

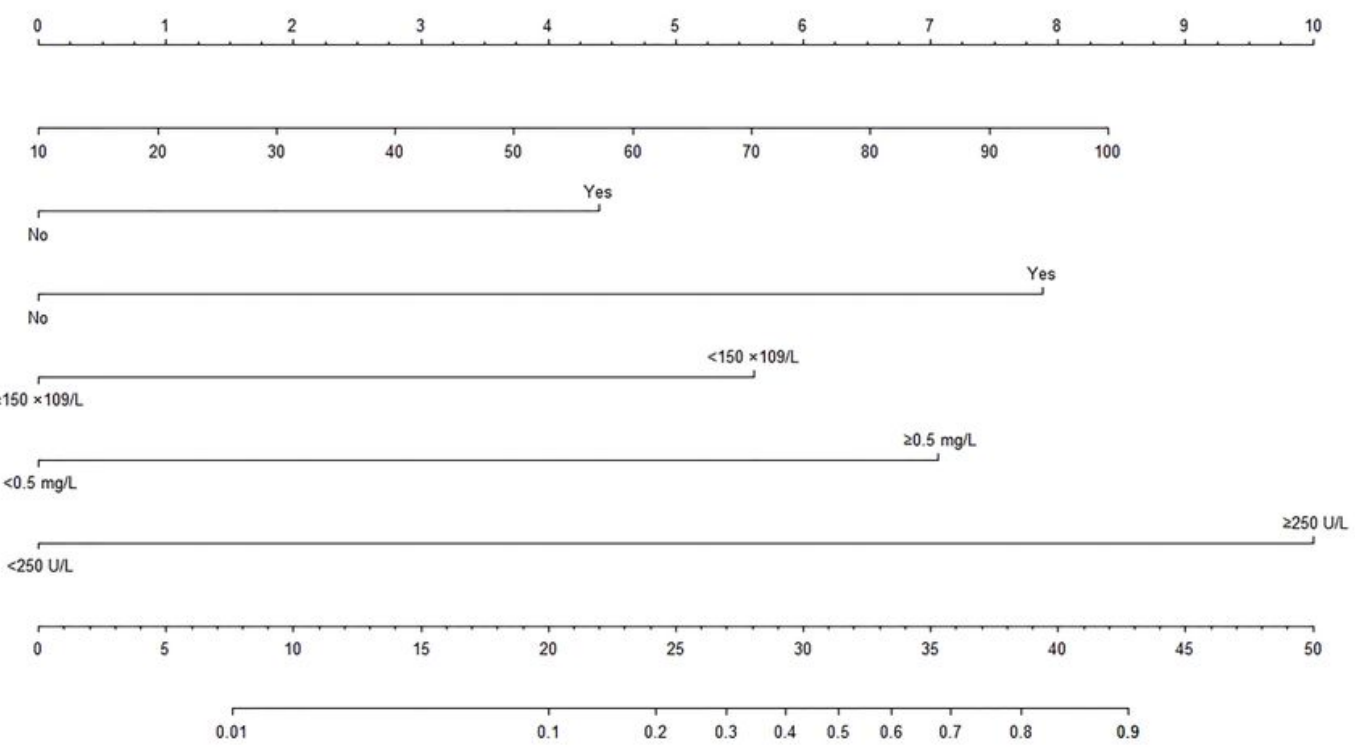

\section{Figure 3}

Nomogram for severity of COVID-19 at admission in the development cohort. To use the nomogram, an individual patient's value is located on each variable axis, and a line is drawn upward to determine the number of points received for each variable value. The sum of these numbers is located on the Total Points axis, and a line is drawn downward to the "Risk axes" to determine the likelihood of severity of COVID-19 at admission. 


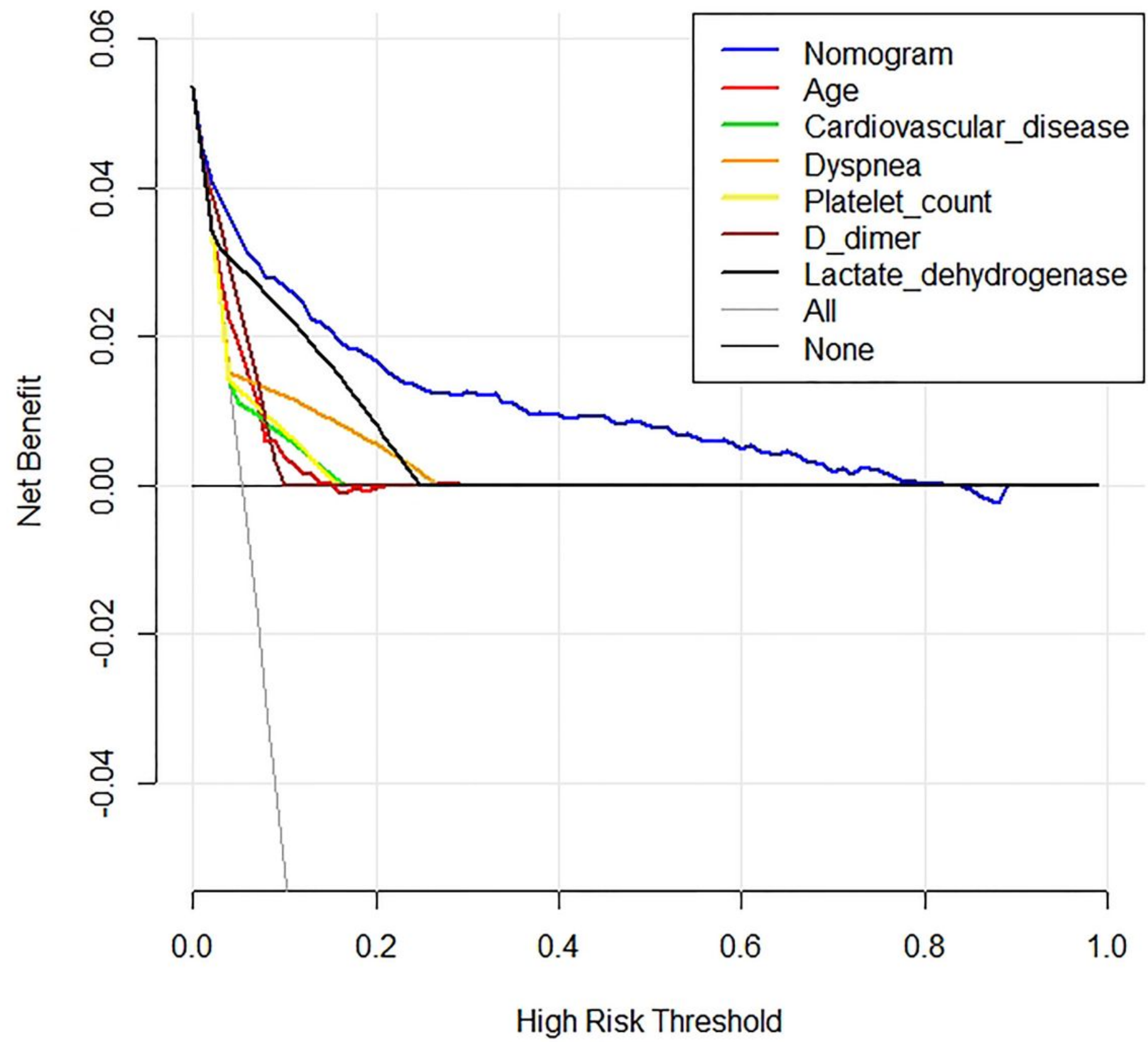

Figure 4

Decision curve analysis for severity of COVID-19 at admission. Decision curve analysis was performed to compare the performance of the nomogram with that of all univariable models.

\section{Supplementary Files}

This is a list of supplementary files associated with this preprint. Click to download. 
- supplementarymaterials.docx 